\title{
EXCITATION OF NUCLEAR COLLECTIVE STATES BY HEAVY IONS WITHIN THE MODEL OF SEMIMICROSCOPIC OPTICAL POTENTIAL
}

\author{
K.M.Hanna ${ }^{a}$, K. V. Lukyanov ${ }^{b}$, V. K. Lukyanov ${ }^{b}$, \\ Z. Metawei ${ }^{c}$, B. Slowinski ${ }^{\text {, e }}$, E. V. Zemlyanaya ${ }^{b}$ \\ ${ }^{a}$ Mathematical and Theoretical Physics Department, NRC, Atomic Energy Authority, Cairo, Egypt \\ ${ }^{b}$ Joint Institute for Nuclear Research, Dubna \\ ${ }^{c}$ Physics Department, Faculty of Science, Cairo University, Giza, Egypt \\ ${ }^{d}$ Faculty of Physics, Warsaw University of Technology, Warsaw, Poland \\ ${ }^{e}$ Institute of Atomic Energy, Otwock-Swierk, Poland
}

The (semi)microscopic double-folding nucleus-nucleus optical potentials are suggested for consideration of inelastic scattering with excitation of collective nuclear states by using the adiabatic approach and the elastic scattering amplitude in the high-energy approximation. The analytical expression for inelastic scattering amplitude is obtained keeping the first-order terms in the deformation parameter of a potential. Calculations of inelastic cross sections for the ${ }^{17} \mathrm{O}$ heavy ions scattered on different nuclei at about hundred $\mathrm{MeV} /$ nucleon are made, and the acceptable qualitative agreement with the experimental data is obtained without introducing free parameters. The prospect of the method for further applications is discussed.

Для рассмотрения неупругого рассеяния с возбуждением коллективных состояний ядер предлагается использовать (полу)микроскопический ядро-ядерный оптический потенциал, адиабатический подход и амплитуду упругого рассеяния в высокоэнергетическом приближении. Получено аналитическое выражение для амплитуды неупругого рассеяния, где удерживаются члены первого порядка в разложении потенциала по параметру деформации. Выполнены расчеты неупругих сечений рассеяния тяжелых ионов ${ }^{17} \mathrm{O}$ разными ядрами при энергиях около 100 МэВ/нуклон, и получено удовлетворительное согласие с экспериментальными данными без введения свободных параметров. Обсуждаются возможности предложенного подхода для дальнейших приложений.

\section{INTRODUCTION}

The theory of excitations of nuclear collective states in peripheral nuclear collisions is based on the elastic scattering optical potential $U(r)=V(r)+i W(r)$. This latter is used to obtain the transition potential $U_{\text {int }}=U_{\text {int }}^{(N)}+U_{\text {int }}^{(C)}$ for inelastic channel. Recently, in [1], the nucleus-nucleus inelastic scattering with excitation of $2^{+}$rotational states was considered in the framework of the high-energy approximation utilizing the phenomenological WoodsSaxon type potential. The collective variables $\left\{\alpha_{\lambda \mu}\right\}$, which characterize the deformation of the surface of a potential, were introduced through the radius

$$
\Re=R+\delta R, \quad \delta R=R \sum_{\lambda \mu} \alpha_{\lambda \mu} Y_{\lambda \mu}(\theta, \phi) .
$$


Here $\theta, \phi$ are spherical coordinates of a space vector $\mathbf{r}$ in the laboratory system. The wave functions of rotational states and collective variables $\left\{\alpha_{\lambda_{\mu}}\right\}$ are given as follows:

$$
|I M\rangle=\sqrt{\frac{2 I+1}{8 \pi^{2}}} D_{M 0}^{(I)}\left(\Theta_{i}\right), \quad \alpha_{2 \mu}=\beta_{2} D_{\mu 0}^{(2) *}\left(\Theta_{i}\right),
$$

where $\beta_{2}$ is the deformation parameter and $\left\{\Theta_{i}\right\}$ are the intrinsic axis rotational angles.

In [1], suggesting small $\beta_{2} \ll 1$, the transition potential was obtained as the derivative of $U(r, \Re)$, and the inelastic scattering amplitude was derived in adiabatic approximation

$$
f_{I M}(q)=\left\langle I M\left|f\left(q,\left\{\alpha_{\lambda \mu}\right\}\right)\right| 00\right\rangle
$$

where $q=2 k \sin (\vartheta / 2)$ is the momentum transfer; $k$ is the relative momentum, and $\vartheta$, the angle of scattering. The elastic scattering amplitude $f\left(q,\left\{\alpha_{\lambda \mu}\right\}\right)$ was taken in the highenergy approximation (HEA) with the «frozen» coordinates of collective motion $\left\{\alpha_{\lambda \mu}\right\}$. Then, inelastic cross sections for the ${ }^{17} \mathrm{O}$ heavy ions scattered on different nuclei at about hundred $\mathrm{MeV} /$ nucleon were calculated, and an acceptable agreement with the experimental data was received. So, the conclusions were made on applicability of HEA to study the nucleus-nucleus inelastic processes.

The aim of this paper is to apply not phenomenological but microscopic potentials for calculating an inelastic scattering amplitude. The matter of fact is that the phenomenological potentials, used for inelastic scattering, must be specially fitted in the corresponding elastic channel at the same energy and for the same couple of scattered nuclei as they are in inelastic channel. Otherwise, at present there are no tables of global optical potentials for the heavyion elastic scattering at different energies and kinds of colliding nuclei. Moreover, there exists the problem of ambiguity of parameters of phenomenological potentials (see, e.g., [2]) since the fit needs a large amount of data, and thus any additional information, involved into consideration, in particular, the data of inelastic scattering, is very desirable.

On the other hand, in the last two decades of years the double-folding (DF) microscopic nucleus-nucleus potentials occur rather popular. They are calculated using the following expression:

$$
\begin{aligned}
& V^{\mathrm{DF}}(r)=V^{D}(E, r)+V^{\mathrm{EX}}(E, r)=\int d^{3} r_{1} d^{3} r_{2} \rho_{1}\left(\mathbf{r}_{1}\right) \rho_{2}\left(\mathbf{r}_{2}\right) v^{D}\left(\rho, E, r_{12}\right)+ \\
&+\int d^{3} r_{1} d^{3} r_{2} \rho_{1}\left(\mathbf{r}_{1}, \mathbf{r}_{1}+\mathbf{r}_{12}\right) \rho_{2}\left(\mathbf{r}_{2}, \mathbf{r}_{2}-\mathbf{r}_{12}\right) v^{\mathrm{EX}}\left(\rho, E, r_{12}\right) \exp \left[\frac{i \mathbf{k}(\mathbf{r}) \mathbf{r}_{12}}{M}\right]
\end{aligned}
$$

where $\mathbf{r}_{12}=\mathbf{r}+\mathbf{r}_{2}-\mathbf{r}_{1}$ is the distance between nucleons of colliding nuclei; $k(r)$ is the local momentum of relative motion of nuclei, and $M=A_{1} A_{2} /\left(A_{1}+A_{2}\right)$. (For details see, e.g., [3, 4].) These DF potentials apply the nuclear density distributions $\rho(\mathbf{r})$ and matrices $\rho(\mathbf{x}, \mathbf{y})$, and also include the effective nucleon-nucleon potentials $v^{D}$ and $v^{\mathrm{EX}}$. In principle, all of these quantities are known from independent experimental studies. Dependence of $N N$ potentials on kinetic energy and on the matter density in overlapping region of nuclei was also established. DF potentials take into account the antisymmetrization of the system by accounting for the knock-on effects (interchange of nucleons 1 and 2) and describe sufficiently well the shape of the peripheral region of potentials, very important in formations of both the differential and total cross sections. For a period of years, in comparison with experimental data, these 
real DF potentials were supplemented by the phenomenological imaginary potentials $W^{P}(r)$ having three (or more) free parameters. By doing so, it was shown that one needs to diminish slightly the calculated real part by introducing a renormalization coefficient $N_{r}$, and thus, the whole potential $U(r)=N_{r} V^{\mathrm{DF}}+i W^{P}(r)$ has four (or more) free parameters.

However, recently in [5], it was demonstrated that the imaginary part can also be calculated microscopically by transforming the eikonal phase of the high-energy microscopical theory [6, 7] of scattering of complex systems. It was shown in [5] that this imaginary potential $W^{H}$ contains the folding integral which corresponds to the integral of only the direct part $V^{D}$ of the DF potential (4). This optical potential has the form $U(r)=N_{R} V^{\mathrm{DF}}(r)+i N_{\mathrm{im}} W^{H}(r)$. In addition, it was reasonable to generalize this form to include the exchange term, too, and then to test the potential $U(r)=N_{r} V^{\mathrm{DF}}+i N_{\mathrm{im}} V^{\mathrm{DF}}(r)$, as well. These potentials were called the semimicroscopic ones because their basic forms $W^{H}$ and $V^{\mathrm{DF}}$ were calculated microscopically, without introducing free parameters, and only two parameters $N_{r}$ and $N_{\mathrm{im}}$ must be adjusted to experimental data.

Figure 1 shows by dashed lines the double-folding potentials $V^{\mathrm{DF}}$ calculated in [8] for scattering of the ${ }^{17} \mathrm{O}$ heavy ions on different nuclei at $E_{\text {lab }}=1435 \mathrm{MeV}$. The respective optical potentials were adjusted to the elastic scattering differential cross sections using for
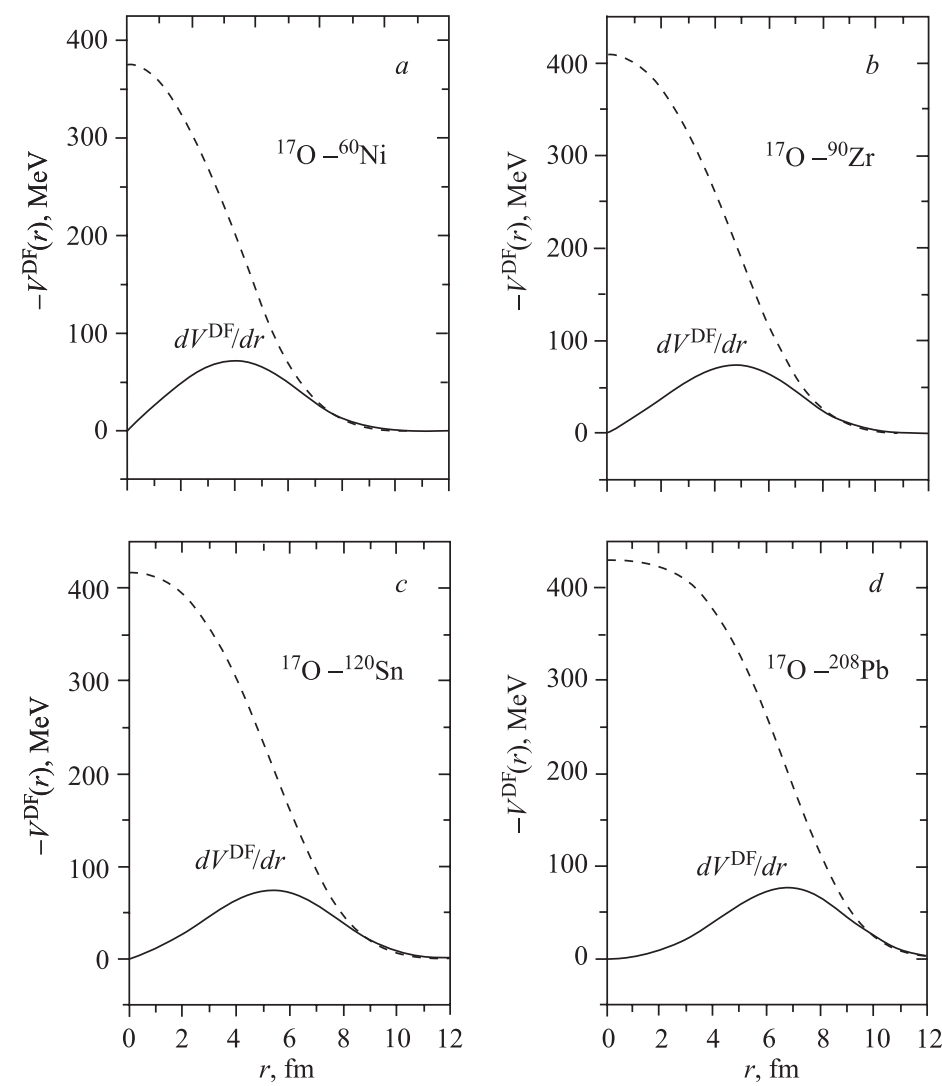

Fig. 1. The double-folding potentials (dashed lines) and their derivatives (solid lines) calculated for different couples of nuclei at $E_{\text {lab }}=1435 \mathrm{MeV}$ 

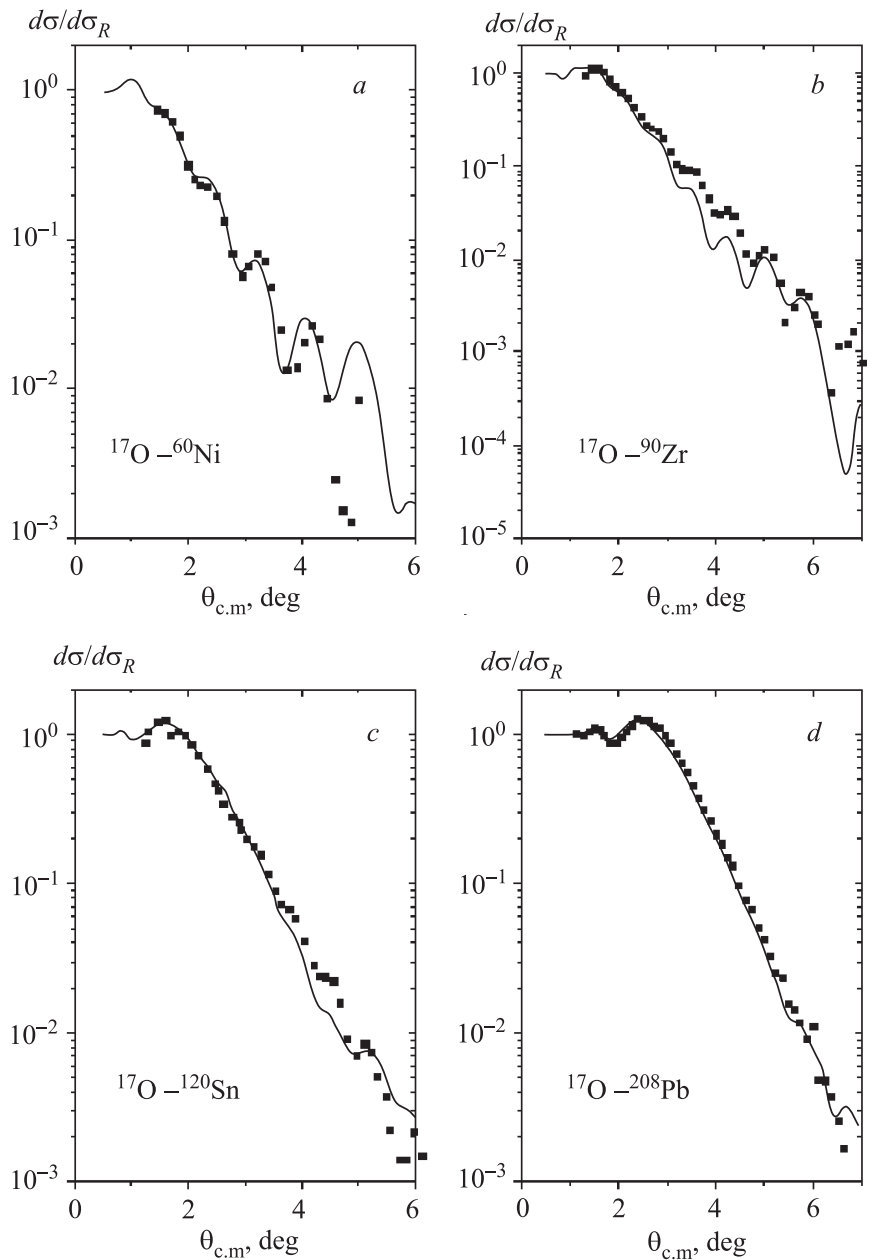

Fig. 2. The ratio of the elastic scattering differential cross sections to the Rutherford one (solid lines) calculated using the semimicroscopic optical potentials $N_{r} V^{\mathrm{DF}}+i N_{\mathrm{im}} V^{\mathrm{DF}}$ at $E_{\mathrm{lab}}=1435 \mathrm{MeV}$ and compared with the experimental data from [9]

imaginary terms the forms $N_{\mathrm{im}} W^{H}$ and $N_{\mathrm{im}} V^{\mathrm{DF}}$. In Fig. 2 we reproduce the ratios of elastic cross sections to the Rutherford one calculated in [8] in the framework of the high-energy approximation using the microscopic potentials $U(r)=N_{r} V^{\mathrm{DF}}(r)+i N_{\mathrm{im}} V^{\mathrm{DF}}$, and their comparisons with the experimental data from [9]. The adjusted normalization coefficients $N_{r}$ and $N_{\text {im }}$ were obtained as 0.6 and 0.6 for ${ }^{60} \mathrm{Ni}, 0.6$ and 0.5 for ${ }^{90} \mathrm{Zr}, 0.5$ and 0.5 for ${ }^{120} \mathrm{Sr}$, and 0.5 and 0.8 for ${ }^{208} \mathrm{~Pb}$. One sees fairly well agreement with the data in the region of an applicability of HEA at $\theta \leqslant \sqrt{2 / k R}$. So, these potentials can be applied further in calculations of inelastic scattering of the same nuclei at the same energy for comparisons with existent experimental data on the $2^{+}$state excitations [9]. 


\section{SOME FORMULAE AND COMMENTS}

The microscopic potentials have no obvious parameters something like the radius $R$ and diffuseness $a$ of a Woods-Saxon potential. Therefore, in order to introduce there the dependence on internal collective variables $\alpha_{\lambda \mu}$, we make, in analogy with (1), the respective changes of spatial coordinates

$$
r \Rightarrow r+\delta r, \quad \delta r=-r \sum_{\lambda \mu} \alpha_{\lambda \mu} Y_{\lambda \mu}(\theta, \phi) .
$$

Then, expanding the potential in $\delta r$ we obtain the generalized optical potential consisting of two terms, the spherically symmetrical and deformed one:

$$
U^{(N)}\left(r,\left\{\alpha_{\lambda \mu}\right\}\right)=U^{(N)}(r)+U_{\text {int }}^{(N)}\left(r,\left\{\alpha_{\lambda \mu}\right\}\right),
$$

where the transition potential (its nuclear part) is as follows:

$$
U_{\mathrm{int}}^{(N)}=-r \frac{d}{d r} U(r) \sum_{\mu} \alpha_{2 \mu} Y_{2 \mu}(\theta, \phi) .
$$

In Fig. 1 one can see the behavior of the derivatives of microscopic potentials $V^{\mathrm{DF}}$ for the above-considered cases. All of them have typical maxima in the surface region of a potential. The respective quadrupole part of the generalized Coulomb potential $U^{(C)}\left(r,\left\{\alpha_{\lambda \mu}\right\}\right)$ is obtained as usually with the help of its definition through the uniform charge density distribution having the radius $\Re$ as in (1) with $R_{C}=r_{c}\left(A_{1}^{1 / 3}+A_{2}^{1 / 3}\right)$. This yields in [1]

$$
U_{\mathrm{int}}^{(C)}=\frac{3}{5} U_{B}\left[\left(\frac{r}{R_{C}}\right)^{2} \Theta(R-r)+\left(\frac{R_{C}}{r}\right)^{3} \Theta(r-R)\right] \sum_{\mu} \alpha_{2 \mu} Y_{2 \mu}(\theta, \phi),
$$

where $U_{B}=Z_{1} Z_{2} e^{2} / R_{C}$.

Then, we use the expression for high-energy amplitude of scattering

$$
f(q)=i \frac{k}{2 \pi} \int b d b d \phi \mathrm{e}^{i q b \cos \phi}\left[1-\mathrm{e}^{i \Phi}\right] .
$$

Here integration is performed over impact parameters $b$ and on its azimuthal angle $\phi$, and the eikonal phase is determined by the nucleus-nucleus potential

$$
\Phi=-\frac{1}{\hbar v} \int_{-\infty}^{\infty} U(r+\delta r) d z, \quad r=\sqrt{b^{2}+z^{2}}
$$

where $v$ is the relative velocity of colliding nuclei. Substituting here the total potential having the central and transition terms, one can write

$$
\begin{gathered}
\Phi=\Phi_{0}(b)+\Phi_{\text {int }}\left(b,\left\{\alpha_{\lambda \mu}\right\}, \phi\right), \\
\Phi_{\text {int }}=\beta_{2} \sum_{\mu=0, \pm 2} G_{\mu}(b) D_{\mu 0}^{(2) *}\left(\Theta_{i}\right) \mathrm{e}^{i \mu \phi},
\end{gathered}
$$




$$
\begin{aligned}
G_{\mu}(b)=-\frac{2}{\hbar v} \int_{0}^{\infty} d z Y_{2 \mu}(\arccos (z / r), 0) \times \\
\times\left[-r \frac{d U(r)}{d r}+\frac{3}{5} U_{B}\left[\left(\frac{r}{R_{C}}\right)^{2} \Theta(R-r)+\left(\frac{R_{C}}{r}\right)^{3} \Theta(r-R)\right]\right],
\end{aligned}
$$

where $r=\sqrt{b^{2}+z^{2}}$. Substituting (11) in (9) and (3), and expanding the exponential function $\exp \left(i \Phi_{\text {int }}\right)$, we retain only a term of the first order in $\beta_{2}$. Then, integration over rotational angles $\Theta_{i}$ can be performed, and one gets the inelastic scattering amplitudes $f_{\lambda \mu}(q)$ and differential cross section as follows [1]:

$$
\begin{gathered}
f_{20}(q)=\frac{k}{\sqrt{5}} \beta_{2} \int_{0}^{\infty} b d b J_{0}(q b) G_{0}(b) \mathrm{e}^{i \Phi_{0}(b)}, \\
f_{22}(q)=-\frac{k}{\sqrt{5}} \beta_{2} \int_{0}^{\infty} b d b J_{2}(q b) G_{2}(b) \mathrm{e}^{i \Phi_{0}(b)}, \\
\frac{d \sigma_{\text {in }}}{d \Omega}=\left|f_{20}\right|^{2}+2\left|f_{22}(q)\right|^{2} .
\end{gathered}
$$

\section{COMPARISON WITH EXPERIMENTAL DATA. SUMMARY}

When calculating the elastic and inelastic scattering amplitudes one has to take into account the Coulomb distortion of the straight-ahead trajectory situated in expressions of the highenergy theory. This is made by exchanging, in the nuclear part of the phases $\Phi_{0}(b)$ and $\Phi_{\text {int }}(b)$, the impact parameter $b$ by the distance of the turning point in the Coulomb field of the point charge, i.e., $b \Rightarrow b_{c}=\bar{a}+\sqrt{b^{2}+\bar{a}^{2}}$, where $\bar{a}=Z_{1} Z_{2} e^{2} / \hbar v k$ is a half of closest approach distance at $b=0$.

Firstly, we estimate inelastic cross sections of scattering of ${ }^{17} \mathrm{O}$ on different nuclei without introducing any free parameters. To this end, we apply semimicroscopic optical potentials $U=N_{r} V^{\mathrm{DF}}+i N_{\mathrm{im}} V^{\mathrm{DF}}$ calculated and adjusted in [8] to the experimental data on elastic scattering of the same nuclei [9]. The deformation parameters $\beta_{2}^{(n)}$ and $\beta_{2}^{(c)}$ for nuclear and Coulomb potentials, separately, were suggested to obey the relation $\beta_{2}^{(c)} \bar{R}_{C}=\beta_{2}^{(n)} \bar{R}_{n}$, where $\bar{R}$ are rms radii. Qualitatively, this relation supposes an equality of areas of rings on the $r$ plane, where the main transition takes place. The $\beta_{2}^{(c)}$ deformations are taken as they were extracted in [9] using the known reduced electric transition probabilities $B(E 2 \uparrow)$ in the target nuclei. (For parameters see set 1 in the table.)

Figure 3 shows these results by dashed lines. We see that the calculations performed without free parameters are in a qualitative agreement with the experimental data. The slopes of all curves are in coincidence with the behavior of the data. As to the absolute values of cross sections, they can be slightly improved by increasing the deformation parameters. An exception is seen at small angles (very peripheral collisions) for heavy nuclei ${ }^{120} \mathrm{Sn},{ }^{208} \mathrm{~Pb}$ (large charges), where the multistep Coulomb excitation must give large contribution while 
Deformation parameters of the Coulomb and nuclear potentials

\begin{tabular}{|c|c|c|c|c|c|}
\hline & $\beta_{2}$ & ${ }^{17} \mathrm{O}+{ }^{60} \mathrm{Ni}$ & ${ }^{17} \mathrm{O}+{ }^{90} \mathrm{Zr}$ & ${ }^{17} \mathrm{O}+{ }^{120} \mathrm{Sn}$ & ${ }^{17} \mathrm{O}+{ }^{208} \mathrm{~Pb}$ \\
\hline \multirow{2}{*}{ Set 1 } & $\beta_{2}^{(c)}$ & 0.2067 & 0.091 & 0.1075 & 0.0544 \\
& $\beta_{2}^{(n)}$ & 0.2453 & 0.1072 & 0.1270 & 0.0644 \\
\hline \multirow{2}{*}{ Set 2 } & $\beta_{2}^{(c)}$ & 0.2067 & 0.091 & 0.1075 & 0.0544 \\
& $\beta_{2}^{(n)}$ & 0.4 & 0.16 & 0.25 & 0.1 \\
\hline
\end{tabular}
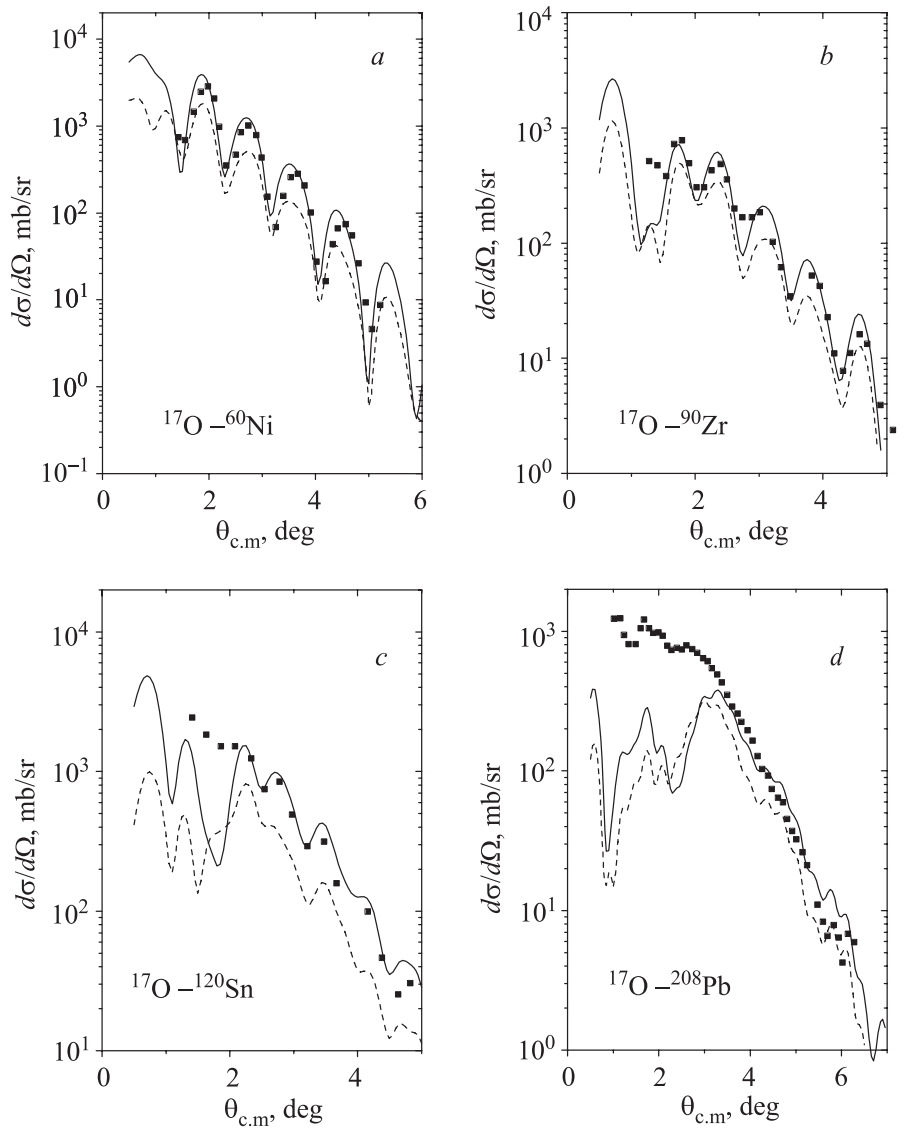

Fig. 3. Microscopic calculations of inelastic scattering differential cross sections of the ${ }^{17} \mathrm{O}$ heavy ions at $1435 \mathrm{MeV}$ on different target nuclei with excitations of $2^{+}$collective states. Dashed curves are for the consistent deformation parameters of the nuclear and Coulomb potentials (set 1 in the table), solid curves - with the free $\beta_{2}^{(n)}$ parameters (set 2). The data are from [9]

in our consideration we take into account only the first power terms of deformations $\beta$ in amplitudes.

Meanwhile, if we refuse to fulfill the above relation between $\beta_{2}^{(c)}$ and $\beta_{2}^{(n)}$, and suppose the deformation of nuclear potential $\beta_{2}^{(n)}$ to be free parameter (solid curves), then agreements 
with the data become fairly better as compared to the preceding calculations. (For parameters see set 2 in the table.)

Summarizing the obtained results, we note that, first, the outlook for the further work in utilizing (semi)microscopic potentials, both the real and the imaginary one, for parametrization and analysis of experimental data is rather attractive. Their applications do not need to introduce so many parameters as they included in the case of phenomenological potentials.

Second, our consideration was based on the simple adiabatic approximation and utilizing only the linear terms, in $\beta_{2}$, of the scattering amplitude. But these potentials can also be applied in the more proper coupled channel method where, in principle, all powers of terms with $\beta_{2}$ are taken into account. By the way, the prospects also exist of improving the suggested approach by constructing the microscopic transition potentials rather than that obtained above from the microscopic potentials themselves.

Acknowledgements. V.K.L. and B.S. acknowledge the support of the Infeld-Bogoliubov Program. E. V.Z. and K. V.L. acknowledge the support of the Russian Foundation for Basic Research (project 06-01-00228).

\section{REFERENCES}

1. Lukyanov V. K., Metawei Z., Zemlyanaya E. V. High-energy approach for heavy-ion scattering with excitations of nuclear collective states. nucl-th/0508015. 2005. 9 p.; Phys. At. Nucl. (submitted).

2. Satchler G. R. Direct Nuclear Reactions. Oxford: Clarendon, 1983. 833 p.

3. Dao Tien Khoa, Knyaz'kov O.M. Exchange effects in nucleus-nucleus potentials and nuclear rainbow scattering // Part. Nucl. 1990. V.21, No.6. P. 1456-1498.

4. Khoa D.T., Satchler G.R. Generalized folding model for elastic and inelastic nucleus-nucleus scattering using realistic density dependent nucleon-nucleon interaction // Nucl. Phys. A. 2000. V.668. P. 3-41.

5. Lukyanov V.K., Zemlyanaya E. V., Lukyanov K. V. Nucleus-nucleus scattering in the high-energy approximation and optical folding potential. JINR Preprint P4-2004-115. Dubna, 2004. 16 p.; Phys. At. Nucl. 2006. V.69, No. 2. P. 240-254.

6. Glauber R. J. Lectures in Theoretical Physics. N. Y., 1959. P. 315.

7. Sitenko A. G. // On the theory of nuclear reactions involving complex particles // Ukr. Fiz. Zh. 1959. V.4. P. $152-163$.

8. Hanna K. M. et al. Restoration of heavy-ion potentials at intermediate energies // Proc. of the 4th Conf. on Nucl. Part. Phys., Fayoum, Egypt. Cairo, 2004. P. 26-36; nucl-th/0410015. 2004. 11 p.

9. Neto R. L. et al. Excitation of giant resonances in ${ }^{208} \mathrm{~Pb},{ }^{120} \mathrm{Sn},{ }^{90} \mathrm{Zr}$ and ${ }^{60} \mathrm{Ni}$ by $84 \mathrm{MeV} /$ nucleon ${ }^{17}$ O ions // Nucl. Phys. A. 1993. V. 560. P. 733-764. 